\title{
Aporte del IVA a la economía del Ecuador e influencia en la generación de nuevos proyectos de inversión
}

\section{VAT contribution to the economy of Ecuador and influence in the generation of new investment projects}

Lenin Agustín Chamba Bastidas. ${ }^{1}$, Raquel Virginia Colcha Ortiz. ${ }^{2}$, María del Carmen Moreno Albuja. ${ }^{3}$, Marco Antonio Gavilanes Sagñay. ${ }^{4}$

\section{DOI: https://doi.org/10.33262/visionariodigital.v3i3.889}

\begin{abstract}
Taxes play an important role in the growth and development of individuals in a given country, since they infringe on micro as in macroeconomics, they effectively serve as one of the main tools in what regards fiscal policy in general terms.

When we refer to the Value Added Tax - VAT, we are referring to a tax called indirect and regressive, since not everyone pays for their taxable capacity or income level as if it happens with direct taxes such as Tax Rent.

The distribution of VAT to the total collection is around $50 \%$ with projections to be maintained when it is appropriate to raise the collection of direct tax and lower the contribution of VAT, a situation that is not observed in the analyzed data. VAT is a tax that is ultimately paid by final consumers, whether they are high or low income or economic conditions, in the event that the rate of the good or service is $12 \%$ and not $0 \%$, in monetary terms, The payment on the same tax base is the same value for both strata.
\end{abstract}

Keywords: Value Added Tax, Economy, Projects.

\section{Resumen}

Los impuestos cumplen un papel importante en el crecimiento y desarrollo de los individuos de un determinado país, ya que influyen en la micro como en la macroeconomía, sirven de manera efectiva como una de las herramientas principales en lo que respecta a política fiscal en términos generales.

Cuando nos referimos al Impuesto al Valor Agregado - IVA, nos estamos refiriendo a un impuesto denominado indirecto y regresivo, ya que no todos pagan en función a su capacidad

\footnotetext{
${ }^{1}$ Escuela Superior Politécnica de Chimborazo, Facultad de Administración de Empresas, Riobamba, Ecuador, lenin.chamba@espoch.edu.ec

${ }^{2}$ Escuela Superior Politécnica de Chimborazo, Facultad de Administración de Empresas, Riobamba, Ecuador, raquel.colcha@espoch.edu.ec

${ }^{3}$ Escuela Superior Politécnica de Chimborazo, Facultad de Administración de Empresas, Riobamba, Ecuador, Carmen.moreno@espoch.edu.ec

${ }^{4}$ Escuela Superior Politécnica de Chimborazo, Facultad de Administración de Empresas, Riobamba, Ecuador, marco.gavilanes@espoch.edu.ec
} 
contributiva o a su nivel de ingresos como si sucede con los impuestos directo como el Impuesto a la Renta.

La contribución del IVA al total de recaudación está alrededor del 50\% con proyecciones a mantenerse cuando lo adecuado es que suba la recaudación del impuesto directo y baje el aporte del IVA, situación que no se observa en la data analizada.

El IVA es un impuesto que al final lo terminan pagando los consumidores finales, sean estos de altos o bajos ingresos o condiciones económicas, en el evento que la tarifa del bien o servicio sea del $12 \%$ y no del $0 \%$, en términos monetarios, el pago sobre la misma base imponible, es el mismo valor para ambos estratos.

Palabras Claves: Impuesto al Valor Agregado, Economía, Proyectos.

\section{Introducción}

Países como el Ecuador que aún están en un proceso de ingresar a los sectores económicos industrial y de servicios y que tienen como fuente importante en la generación de recursos al sector primario, es necesario analizar de manera frecuente la evolución de la recaudación de impuestos del Estado central, que en nuestro caso son administrados por el Servicio de Rentas Internas - SRI.

En la información que brinda el SRI en la rendición de cuentas del año 2018, se observa en términos generales una tendencia al crecimiento en la recaudación de impuestos en lo que va desde el año 2000 al 2018 con un leve decrecimiento en el año 2016 y 2017 que en lo posterior se recupera en el año 2018, recuperación que también obedece al proceso de la remisión tributaria, proceso de mucha importancia para el análisis debido a su focalización en el beneficio directo que tuvieron las personas naturales y jurídicas que se alinearon y que será motivo de un estudio posterior al presente.

El impuesto al valor agregado - IVA es considerado como un impuesto indirecto y regresivo, esto significa que su tarifa es constante independientemente del nivel de ingresos del sujeto pasivo sobre quién recae dicho impuesto, lo cual es motivo de análisis debido a que es uno impuesto que aporta con un 50\% aproximadamente a la recaudación total de impuestos que administra el SRI.

Desde el punto de vista de la recaudación tal cual, es un aporte significativo en los ingresos del Estado para efectos de cubrir las necesidades, no obstante, hay un impacto en la sociedad tanto por el pago de IVA que realizan los sujetos pasivos, cuanto lo que reciben de manera general como respuesta del Estado quién administra dichos recursos, en especial aquellas personas que están consideradas como pobres o dentro de los límites de pobreza extrema en el Ecuador.

Una de las preguntas importantes que está en el ambiente económico tributario es saber si es bueno o malo que se incremente la recaudación en IVA, ya que por una parte se observa en el Art. 300 de la Constitución vigente del Ecuador los principios y la política tributaria, donde se evidencia claramente la progresividad, concepto que no tiene relación directa con el IVA.

\section{Desarrollo}

Los impuestos no son un tema reciente a nivel mundial ni a nivel del Ecuador, es una práctica con un gran cúmulo de historia tanto desde el punto de vista conceptual cuanto desde la práctica como tal, es evidente que en la actualidad la gestión por parte del Estado y por los entes económicos públicos y privados, es más fácil y llevadera debido a las importantes 
herramientas que la ciencia y la tecnología ponen a disposición en el mercado, esto no quita o elimina de completo la evasión y elusión tributara, misma que en algunos casos se presenta por falta de conocimiento y en otros por mucho conocimiento de quienes cometen esta ilícita actividad.

Es probable que el Estado no solamente cumpla con la obtención de ingresos mediante la recaudación de impuestos, sino que también sirva esta actividad para tener controlada a la ciudadanía, a continuación, un extracto al respecto.

En las épocas de la Colonia y de la República, la carga impositiva, además de contribuir una herramienta gubernamental de generación de ingresos, sirvió como instrumento de dominación. (Carrasco, 2012)

Nace interrogantes al respecto, es un instrumento pasado o dicha dominación se presenta también en la actualidad mediante otros mecanismos diferentes a los de aquella época, la inequidad en el pago de impuestos o beneficios tributarios podría ser considerado como un instrumento de dominicación o solo una estructura que debe ser considerada a revisión.

Antes de 1830, cuando el Ecuador aún no se constituía como república, algunos de los impuestos que permitían cumplir con lo indicado en el párrafo superior, fueron los quintos reales, las alcabalas, el almojarifazgo, el estanco de aguardiente y otro importante desde el punto de vista del impacto social ya que llegó a considerar al ser humano como una mercancía de intercambio y es el impuesto de los indios, estas alternativas de impuestos nacieron en conjunto a un acontecimiento histórico para el mundo en todos su ámbitos, que es el descubrimiento de América y la venida de los españoles y la colonización.

El Impuesto al Valor Agregado no es un impuesto que nace en Ecuador como algunas otras cosas, es un impuesto del sistema europeo que es donde no necesariamente nace, pero si se materializa con mayor énfasis que en ostros países como Canadá, por ejemplo.

El artículo 300 de la Constitución vigente del Ecuador, que a continuación lo vamos a citar, describe con claridad los principios tributarios constitucionales y la política tributaria vigente.

Art. 300.- El régimen tributario se regirá por los principios de generalidad, progresividad, eficiencia, simplicidad administrativa, irretroactividad, equidad, transparencia y suficiencia recaudatoria. Se priorizarán los impuestos directos y progresivos.

La política tributaria promoverá la redistribución y estimulará el empleo, la producción de bienes y servicios, y conductas ecológicas, sociales y económicas responsables. (Constitución de la República de Ecuador, 2008)

En consideración al artículo 300 de la máxima norma en el Ecuador, es constitucional la priorización de impuestos directos y progresivos, esto quiere decir que el Estado deberá gestionar en mayor proporción los impuestos directos como el Impuesto a la Renta que si bien a la fecha es muy representativo en su aporte a la recaudación total, no obstante, es discutible y cuestionable el hecho de incrementar la recaudación en términos de impuesto al valor agregado.

Con fecha abril 29 del 2005, se publica el documento denominado Aspectos Sustanciales y Procedimentales de Impuestos al Valor Agregado, documento que rige para todos los países Andinos y puede considerarse como consolidado dentro de sus sistemas tributarios.

En su artículo 1 de este documento y que corresponde al Ámbito de aplicación se describe lo siguiente: 
La presente Decisión regula los aspectos sustanciales y los procedimientos de los impuestos tipo valor agregado que deben armonizarse en los regímenes tributarios de los Países Miembros, a los efectos de facilitar el cumplimiento de los objetivos y compromisos de la Comunidad Andina.

Para todo lo no regulado por esta Decisión se aplicará la normativa interna de cada país. (AGREGADO, 2004)

Más allá de analizar el documento citado, vamos a revisar brevemente la normativa interna del Ecuador ya que es muy claro el acuerdo en el segundo párrafo del artículo 1, lo no regulado por aquel, se aplicará la norma interna, en nuestro caso parte de este marco legal interno es la Ley de Régimen Tributario Interno vigente.

Art. 52.- Objeto del impuesto. - Se establece el Impuesto al Valor Agregado (IVA), que grava al valor de la transferencia de dominio o a la importación de bienes muebles de naturaleza corporal, en todas sus etapas de comercialización, así como a los derechos de autor, de propiedad industrial y derechos conexos; y al valor de los servicios prestados, en la forma y en las condiciones que prevé esta Ley. (LEY DE REGIMEN TRIBUTARIO INTERNO, 2004)

El impuesto al valor agregado se aplica a bienes y servicios con ciertas condiciones y excepciones, no es momento de hacer una análisis más profundo y minucioso sobre los bienes y servicios que aplica y no aplica el impuesto, pero si se quiere tocar brevemente y resaltar sobre todo, que este impuesto lo pagan todos aquellas personas que consumen un bien o servicio que tenga tarifa vigente del $12 \%$, independientemente de su nivel de ingresos, su situación económica, su capacidad contributiva, esto significa que si un empresario compra un producto en 100 dólares, debe pagar 12 dólares de IVA y si una persona pobre compra el mismo producto en los mismos 100 dólares, también debe pagar un impuesto de 12 dólares, por lo tanto este impuesto es altamente regresivo y muy probablemente indeseado por parte de la población que tiene bajos recursos económicos y que no está en su alcance pagar IVA y en otros casos no le alcanza ni para pagar el propio producto.

Para complementar lo dicho en el párrafo anterior, consideramos citar los dos siguientes artículos.

Según el artículo 54 de la Ley de Régimen Tributario Interno vigente en el Ecuador, existen transferencias que nos son objeto de impuesto al valor agregado, lo cual es beneficioso de manera directa para el sujeto pasivo que incurre en uno de aquellos casos y no es conveniente para quién no incurre en los casos, debido a que la recaudación disminuye en la proporción del beneficio, nos atrevemos a decir que las personas de pocos recursos muy difícil que incurran en alguno de estos casos y por ende la posibilidad del beneficio está muy lejos de la realidad.

La tarifa cero a las importaciones que está descrita en el artículo 55 del mismo cuerpo legal, tiene su beneficiario directo que es el importador e indirectamente el consumidor que no paga impuesto por aquellos productos, no obstante, es posible que afecte en debidos casos a la producción interna, afectación que más allá del impuesto se podría materializar en mayor medida por un tema de competitividad.

Para que se materialice el impuesto debe haber un hecho generador, cuya descripción se la deja a continuación.

Art. 16.- Hecho generador. - Se entiende por hecho generador al presupuesto establecido por la ley para configurar cada tributo. 
Art. 17.- Calificación del hecho generador.- Cuando el hecho generador consista en un acto jurídico, se calificará conforme a su verdadera esencia y naturaleza jurídica, cualquiera que sea la forma elegida o la denominación utilizada por los interesados. Cuando el hecho generador se delimite atendiendo a conceptos económicos, el criterio para calificarlos tendrá en cuenta las situaciones o relaciones económicas que efectivamente existan o se establezcan por los interesados, con independencia de las formas jurídicas que se utilicen. (TRIBUTARIO, 2005)

El concepto de hecho generador como otros conceptos relacionados, son muy importantes para comprender la configurar el tributo como tal y todo el proceso que implica el impuesto al valor agregado en el Ecuador.

A partir de este párrafo vamos a revisar algunas variables importantes y que tienen relación directa con el impuesto al valor agregado en especial y con otros impuestos de manera general.

Es procedente pensar que a medida que aumenten la cantidad de sujetos pasivos de impuestos, aumente la recaudación de impuestos por cuanto se entiende que hay más movimientos económico por el incremento de número de contribuyentes, esto solo se puede demostrar con la verificación de la información real, ya que necesariamente debe aumentar el tamaño de la economía para que incremente la recaudación, el incremento por si solo de número de contribuyente no necesariamente permite el incremento de recaudación.

Existen diferentes alternativas válidas a nivel internacional para medir el Producto Interno Bruto, y cada uno de sus componentes tiene una amplia explicación de su variabilidad, tema que no es del presente trabajo, pero lo dejamos señalado en términos generales.

Uno de las alternativas de medición del PIB es el método del gasto que está representado por la siguiente ecuación.

$$
P I B=C+I+G p+(X-M)
$$

Donde:

$\mathrm{C}=$ consumo

$\mathrm{I} \quad=$ inversión

$\mathrm{Gp} \quad=\quad$ gasto público

$\mathrm{X}=$ exportaciones

$\mathrm{M}=$ importaciones

Como se observa en la ecuación el PIB puede variar por la variación de cualquiera de sus componentes, de tal suerte que se requiere un análisis mucho más amplio para definir cuál de estos elementos permitió la variación del PIB en el período que estamos citando.

Sin embargo, las premisas que estamos planteando son relativamente válidas por los datos generales que estamos observado y citando a la vez.

El Producto Interno Bruto - PIB en el Ecuador a precios constantes es como se presente en la siguiente gráfica. 


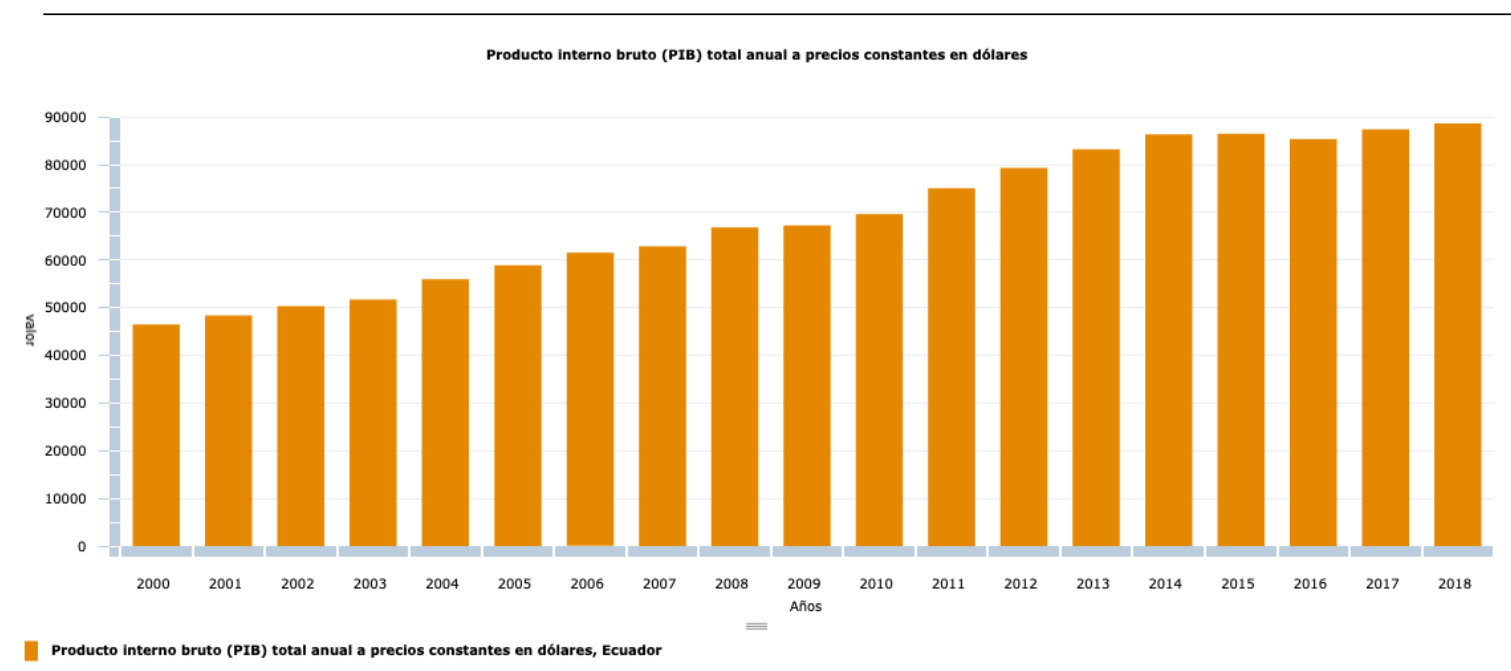

Figura 1: PIB total del Ecuador a precios constantes

Fuente:(http://interwp.cepal.org/cepalstat/engine/index.html, s.f.) - 19-09-2019

Elaborado: Autores

El PIB del Ecuador tiene una tendencia creciente en general con un leve decrecimiento en algunos años y es precisamente el año 2016 donde la diferencia negativa es más representativa y se observa con mayor claridad en la siguiente gráfica.

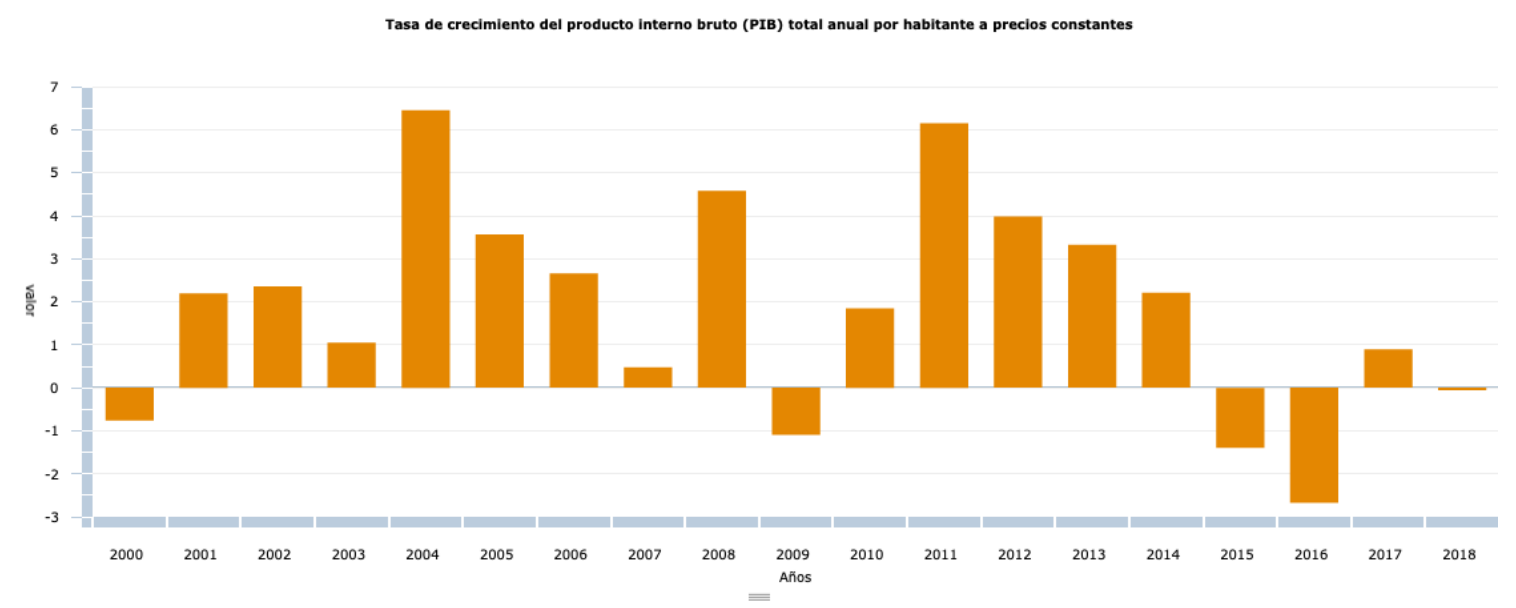

Figura 2: Tasa de crecimiento del PIB del Ecuador a precios constantes

Fuente: (19-09-2019, http://interwp.cepal.org/cepalstat/engine/index.html - 19-09-2019, s.f.) http://interwp.cepal.org/cepalstat/engine/index.html - 19-09-2019

Elaborado: Autores

Una de las premisas planteadas es que la relación del PIB con el número de contribuyentes activos es una relación directamente proporcional y si observamos las dos gráficas podemos decir que la tendencia creciente está en las dos variables, no en las mismas proporciones en el respectivo año, lo que aclara una vez más, el solo hecho de incrementar número de contribuyentes no significa que incremente la recaudación, no es lo mismo que se incremente 
1.000 contribuyentes que en total aportan un millón que se incremente 10 contribuyentes que en total aporten dos millones.

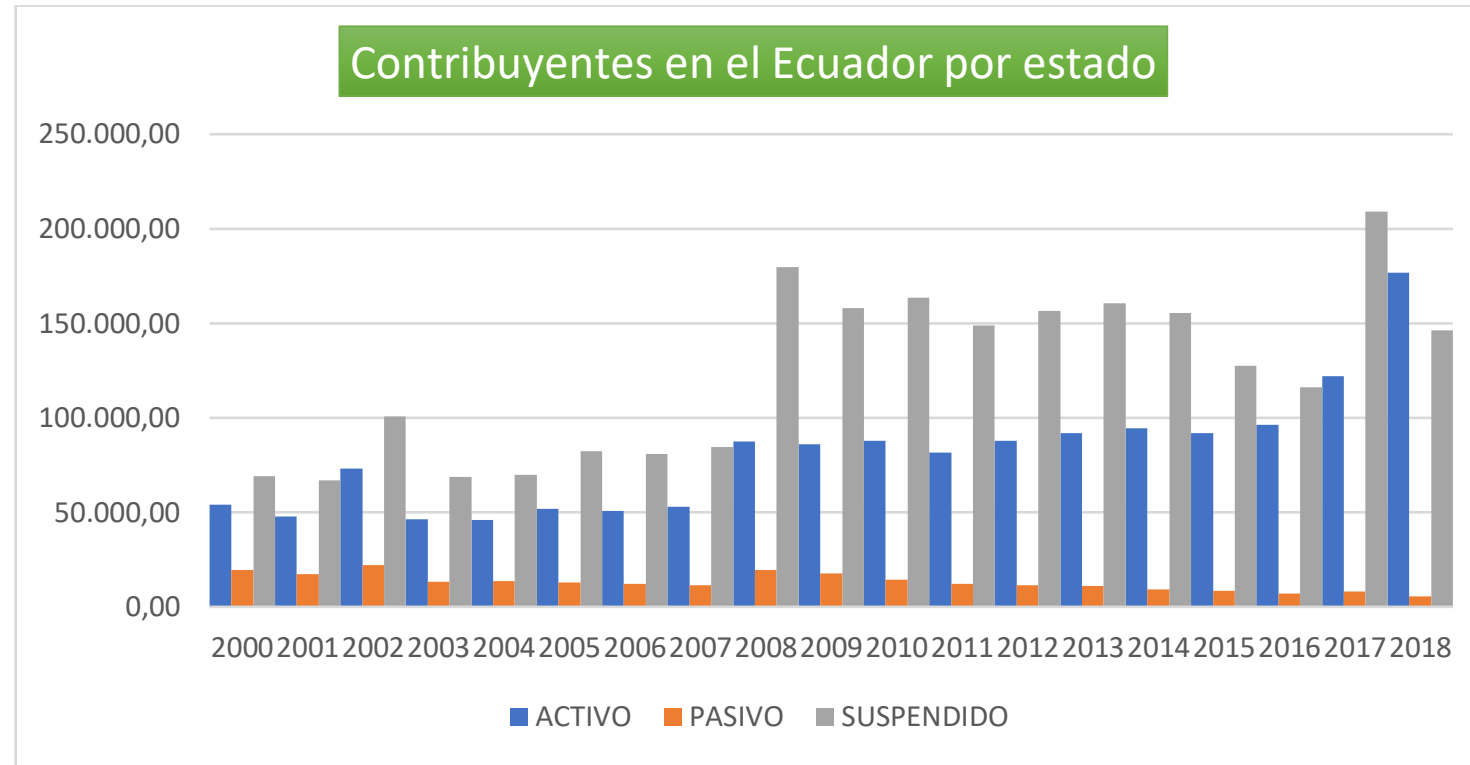

Figura 3: Contribuyentes en el Ecuador por Estado

Fuente: (https://srienlinea.sri.gob.ec/saiku-ui/, s.f.) https://srienlinea.sri.gob.ec/saiku-ui/ Elaborado por: Autores

La recaudación está más enfocada con el tamaño de la economía, y una de las formas de medir es el concepto conocido como presión tributaria si solo queremos medir los tributos de gobierno central o presión fiscal si queremos medir todos los tributos, es oportuno recordar que la política tributaria es una herramienta de la política fiscal y está última de convierte en una herramienta de la política económica, que, entre otros fines, tiene el de encontrar la estabilidad económica.

Es oportuno también observar los datos adicionales que presenta la gráfica 3 y nos referimos a los contribuyentes en estado pasivo y suspendido, estados que pueden mostrar en términos generales temas tan importantes que requieren una investigación profunda como, capitales de corto plazo, quiebra de entes económicos, cambios de país, etc. ya que, si un negocio es rentable para su o sus propietarios, lo más efectivo que pudiera hacer, es mantener activo mi Registro Único d Contribuyentes - RUC.

En la gráfica número 4 que tenemos a continuación, observamos la evolución de la recaudación que va desde el 2000 hasta el año 2018, la cual da una breve idea de lo que es la gráfica del PIB en términos constantes, lo que deja más clara la premisa de que la recaudación depende en gran medida del tamaño de la economía, es decir a mayor economía mayor recaudación, relación que no es lo mismo que la presión tributaria ya que aquella es una relación de la recaudación sobre el PIB y dependerá de algunos factores, como las tarifas de los impuestos, el número de impuestos, la efectividad en la gestión, la cultura de los contribuyentes, entre otros. 


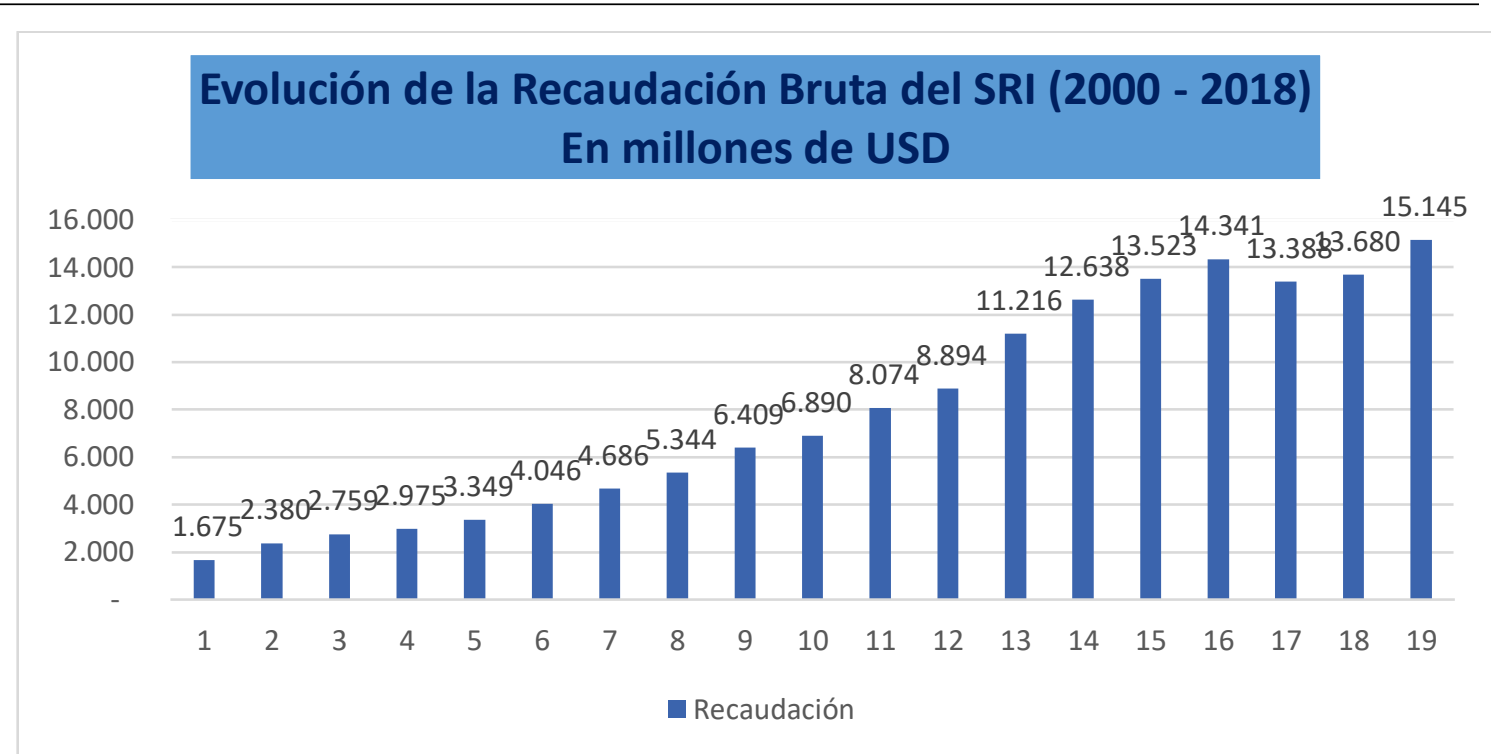

Figura 4: Evolución de la Recaudación bruta del SRI

Fuente: (https://www.sri.gob.ec/web/guest/rendicion-de-cuentas-2018, s.f.)

Elaborado por: Autores

\section{Conclusiones}

El Impuesto al Valor Agregado es un impuesto indirecto altamente regresivo, fácil de recaudar y difícilmente efectivo en el logro de la política tributaria, no permite ejecutar una efectiva redistribución de la riqueza.

$\mathrm{Al}$ aporte que ofrece el IVA, es muy representativo en los ingresos obtenidos por recaudación de impuestos, mismo que está alrededor del 50\% de la participación total, lo que implica un serio problema económico si se dejará de recaudar.

Es un impuesto que a la postre lo terminan pagando los consumidores finales ya que el intermedio se cubre mediante el crédito tributario, a tal punto que los entes económicos significativos con grandes recursos no pagan en función a su capacidad contributiva como si lo hacen en el caso del impuesto a la renta o al menos lo deberían hacer.

Las personas naturales y jurídicas consideradas como inversionistas, analizan una serie de factores para generar proyectos de inversión, su principal objetivo es obtener mayor beneficio posible.

\section{Referencias bibliográficas}

Carrasco, C. M. (2012). Una Nueva Política Fiscal para el Buen Vivir. Quito.

Constitución de la República de Ecuador. (2008). Quito: Registro Oficial . 
Agregado, a. s. (2004). aspectos sustanciales y procedimentales de impuestos valor agregado.

Ley de Régimen Tributario Interno, 1. (2004). Quito: Registro Oficial Suplemento 463.

Tributario, c. (2005). Codigo Tributario. Quito: Registro Oficial Suplemento 38.

http://interwp.cepal.org/cepalstat/engine/index.html. (s.f.). http://interwp.cepal.org/cepalstat/engine/index.html . obtenido de http://interwp.cepal.org/cepalstat/engine/index.html

19-09-2019, h. . (s.f.). Obtenido de http://interwp.cepal.org/cepalstat/engine/index.html 19-09-2019: http://interwp.cepal.org/cepalstat/engine/index.html - 19-09-2019

https://srienlinea.sri.gob.ec/saiku-ui/. (s.f.). Obtenido de https://srienlinea.sri.gob.ec/saikuui/: https://srienlinea.sri.gob.ec/saiku-ui/

https://www.sri.gob.ec/web/guest/rendicion-de-cuentas-2018. (s.f.). Obtenido de https://www.sri.gob.ec/web/guest/rendicion-de-cuentas-2018:

https://www.sri.gob.ec/web/guest/rendicion-de-cuentas-2018

Wikipedia. (09 de 02 de 2008). https://es.wikipedia.org/wiki/Pol\%C3\%ADtica_fiscal.

Recuperado el 09 de 02 de 2008, de Wikipedia:

https://es.wikipedia.org/wiki/Pol\%C3\%ADtica_fiscal

Roura, J. R. (2010). Politica Económica. (C. Sanchez, Ed.) Madrid, España: INTERAMERICANA DE ESPAÑA.

https://www.cepal.org/es. (s.f.). https://www.cepal.org/es. Recuperado el 05 de 01 de 2008, de http://interwp.cepal.org/cepalstat/engine/index.html:

http://interwp.cepal.org/cepalstat/engine/index.html

www.inec.gob.ec. (10 de 02 de 2008). Obtenido de

http://www.ecuadorencifras.gob.ec/base-de-datos-censo-de-poblacion-y-vivienda/\#: http://www.ecuadorencifras.gob.ec/base-de-datos-censo-de-poblacion-y-vivienda/\#

https://www.iadb.org. (15 de 02 de 2008). https://www.iadb.org. Obtenido de https://publications.iadb.org/handle/11319/8601:

https://publications.iadb.org/handle/11319/8601

theglobaleconomy.com. (03 de 01 de 2008). theglobaleconomy.com. Obtenido de http://es.theglobaleconomy.com/Ecuador/Government_size/:

http://es.theglobaleconomy.com/Ecuador/Government_size/

Pavez, P. (2005). Un análisis del IVA en Iberoamérica a través de la experiencia Chilena. Santiago, Chile. 
https://www.sri.gob.ec/web/guest/rendicion-de-cuentas-2018. (s.f.). Obtenido de https://www.sri.gob.ec/web/guest/rendicion-de-cuentas-2018: https://www.sri.gob.ec/web/guest/rendicion-de-cuentas-2018

19-09-2019, h. . (s.f.). http://interwp.cepal.org/cepalstat/engine/index.html - 19-09-2019. Obtenido de http://interwp.cepal.org/cepalstat/engine/index.html - 19-09-2019

\section{LCiencia}




\section{PARA CITAR EL ARTÍCULO INDEXADO}

Chamba Bastidas, L., Colcha Ortiz, R., Moreno Albuja, M., \& Gavilanes Sagñay, M. (2019). Aporte del IVA a la economía del Ecuador e influencia en la generación de nuevos proyectos de inversión. Visionario Digital, 3(3), 337-347.

https://doi.org/10.33262/visionariodigital.v3i3.889

\section{LCiencia}

El artículo que se publica es de exclusiva responsabilidad de los autores y no necesariamente reflejan el pensamiento de la Revista Visionario Digital.

El artículo queda en propiedad de la revista y, por tanto, su publicación parcial y/o total en otro medio tiene que ser autorizado por el director de la Revista Visionario Digital.
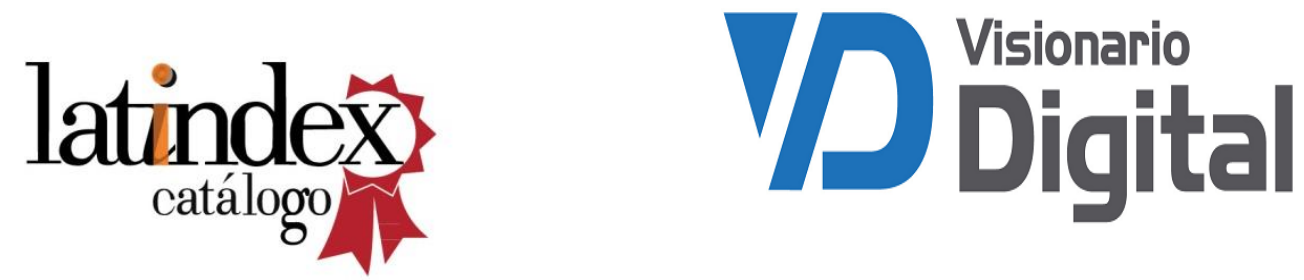